\title{
Geographical heterogeneity in prevalence of subclinical malaria infections at sentinel endemic sites of Myanmar
}

Ziling Liu ${ }^{1,2+}$, Than Naing Soe ${ }^{3 \dagger}$, Yan Zhao ${ }^{1 \dagger}$, Aye Than ${ }^{4}$, Cho Cho ${ }^{4}$, Pyae Linn Aung ${ }^{4}$, Yuling Li ${ }^{1}$, Lin Wang ${ }^{1}$, Huilin Yang ${ }^{1}$, Xiangnan $\mathrm{Li}^{1}$, Danni Li ${ }^{1}$, Zhiping Peng ${ }^{2}$, Jiangang Wang ${ }^{2}$, Yan Li ${ }^{2}$, Zhaoqing Yang ${ }^{5}$, Hongning Zhou ${ }^{6}$, Qinghui Wang ${ }^{1 *}$, Myat Phone Kyaw ${ }^{4^{*}}$, Yaming Cao ${ }^{1 *}$ and Liwang Cui ${ }^{17^{*}}$

\begin{abstract}
Background: The malaria burden of Myanmar still remains high within the Greater Mekong Subregion of Southeast Asia. An important indicator of progress towards malaria elimination is the prevalence of parasite infections in endemic populations. Information about malaria epidemiology is mostly derived from reports of confirmed acute malaria cases through passive case detection, whereas the prevalence of baseline subclinical malaria infections is much less known.

Methods: In this study, cross-sectional surveys were conducted during the rainy season of 2017 in four townships (Bilin, Thabeikkyin, Banmauk and Paletwa) of Myanmar with divergent annual malaria incidences. A total of 1991 volunteers were recruited from local villages and Plasmodium subclinical infections were estimated by light microscopy (LM), rapid diagnostic tests (RDTs) and nested PCR. The nested PCR analysis was performed with a modified pooling strategy that was optimized based on an initial estimate the infection prevalence.

Results: The overall malaria infection prevalence based on all methods was 13.9\% (277/1991) and it differed drastically among the townships, with Paletwa in the western border having the highest infection rate (22.9\%) and Thabeikkyin in central Myanmar having the lowest (3.9\%). As expected, nested PCR was the most sensitive and identified 226 (11.4\%) individuals with parasite infections. Among the parasite species, Plasmodium vivax was the most prevalent in all locations, while Plasmodium falciparum also accounted for $32 \%$ of infections in the western township Paletwa. Two RDTs based on the detection of the hrp2 antigen detected a total of 103 P. falciparum infections, and the ultrasensitive RDT detected 20\% more P. falciparum infections than the conventional RDT. In contrast, LM missed the majority of the subclinical infections and only identified 14 Plasmodium infections.

Conclusions: Cross-sectional surveys identified considerable levels of asymptomatic Plasmodium infections in endemic populations of Myanmar with P. vivax becoming the predominant parasite species. Geographical heterogeneity of subclinical infections calls for active surveillance of parasite infections in endemic areas. The pooling scheme designed for nested PCR analysis offers a more practical strategy for large-scale epidemiological studies of parasite prevalence. Such information is important for decision-makers to put forward a more realistic action plan for malaria elimination.
\end{abstract}

Keywords: Myanmar, Malaria, Prevalence, Subclinical, Pooling strategy

\footnotetext{
* Correspondence: gaihui2010@163.com; kyaw606@gmail.com;

ymcao@cmu.edu.cn; Icui@health.usf.edu

'Ziling Liu, Than Naing Soe and Yan Zhao contributed equally to this work.

'Department of Immunology, College of Basic Medical Sciences, China

Medical University, Shenyang 110122, Liaoning, China

${ }^{4}$ Myanmar Medical Association, Yangon, Myanmar

Full list of author information is available at the end of the article
}

(c) The Author(s). 2019 Open Access This article is distributed under the terms of the Creative Commons Attribution 4.0 International License (http://creativecommons.org/licenses/by/4.0/), which permits unrestricted use, distribution, and reproduction in any medium, provided you give appropriate credit to the original author(s) and the source, provide a link to the Creative Commons license, and indicate if changes were made. The Creative Commons Public Domain Dedication waiver (http://creativecommons.org/publicdomain/zero/1.0/) applies to the data made available in this article, unless otherwise stated. 


\section{Background}

Globally, malaria remains a substantial public health problem and significant economic burden with an estimated 219 million cases and 435,000 deaths in 2017 [1]. Malaria cases and deaths in Southeast Asia accounted for 7 and $6 \%$ of the global burden, respectively. In Southeast Asia, the six countries within the Greater Mekong Subregion (GMS) have a regional goal of malaria elimination by 2030 . To achieve this goal, control efforts to continuously shrink the malarious areas have intensified, whereas in areas where malaria transmission had been interrupted, increased surveillance has been implemented to maintain the malaria-free status and prevent malaria re-introduction [2]. According to the World Malaria Report 2018 [1], the Myanmar malaria burden still remains high within the GMS, and malaria transmission persists in most international border regions. This poses a serious threat to the neighboring countries and greatly hinders regional malaria elimination. Compared to acute malaria cases, asymptomatic malaria infections constitute silent reservoirs of transmission [3]. Therefore, universal coverage of human populations with early diagnosis and effective treatment is essential to reduce morbidity, mortality and transmission. Case detection can be done through passive case detection in hospitals and clinics and active case detection by regular house visits, whereas cross-sectional surveys are conducted to determine the prevalence of infections (mostly asymptomatic). Reports for confirmed malaria cases have shown drastic heterogeneity in malaria distribution, which may also correspond to the prevalence of asymptomatic infections [4-6]. Typically, most malaria infections in endemic areas are asymptomatic, while confirmed cases of acute malaria are just "the tip of an iceberg". Since subclinical infections and complicated transmission patterns can contribute to the persistence of malaria, a reliable estimation of the prevalence of asymptomatic malaria is important to guide and monitor progress toward control and elimination [7].

Conventionally, malaria diagnosis is done by light microscopy (LM), which normally has a detection limit of 10-20 parasites/ $\mu \mathrm{l}$ blood in thick film and 100 parasites $/ \mu \mathrm{l}$ blood in thin film $[8,9]$. In addition, misdiagnosis of the parasite species is common and depends to a large extent on the experience of the microscopist $[8,10]$. Rapid diagnostic tests (RDTs) for antigen detection, although not as technically demanding as LM, also have similar detection limits as LM. Given that a large proportion of subclinical malaria infections have parasite densities below the detectable limit of LM and RDTs, molecular detection methods are required for a more accurate estimate of the overall malaria infection rate. PCR-based methods are the most commonly used molecular assays, which can detect parasites to the genus- or species-specific level [11-14].
However, the use of PCR in epidemiological surveillance is mostly done on a small scale, since the procedure is both laborious and costly. For large-scale surveys, pooling strategies are recommended in order to reduce the cost and time [15-19].

This study aimed to assess malaria prevalence at sentinel sites in Myanmar and provide baseline information for the evaluation of progress toward malaria elimination. Cross sectional surveys were conducted in four townships with different levels of endemicity (from reports of confirmed malaria cases), and parasite infection rates were estimated by using LM, two types of RDTs, and nested PCR incorporating a sample pooling design.

\section{Methods}

\section{Study sites and blood sample collection}

Four Myanmar townships Paletwa (western), Banmauk (northern), Thabeikkyin (central) and Bilin (southeastern) with different malaria transmission intensities were selected to conduct cross-sectional surveys to assess malaria prevalence during the rainy season of 2017 (Fig. 1). The 2016 confirmed malaria cases show that Paletwa had the highest annual incidence rate of malaria ( $>100 \%$ o $P$. falciparum and 10-50\% P. vivax), followed by Banmauk (1-10\% P. falciparum and P. vivax), Bilin (1-10\% P. falciparum and $P$. vivax) and Thabeikkyin $(0.1-1 \%$. $P$. vivax and $P$. falciparum) [20]. Participants were interviewed and information on gender, age, and axillary temperature was recorded. Those with a temperature of $37.3{ }^{\circ} \mathrm{C}$ or above were considered febrile. After consent, finger-prick blood was collected for malaria diagnosis by light microscopy (LM), rapid diagnostic tests (RDTs), and spotted onto filter paper for PCR analysis.

\section{Malaria diagnosis by LM and RDTs}

For LM diagnosis of malaria, Giemsa-stained blood smears were read by microscopists at the field sites following an established standard operating procedure [21]. A slide was considered positive when at least one parasite was found [22, 23]. Two RDTs, SD Bioline malaria Ag $P f$ test (Alere, Yongin-si, Republic of Korea), a conventional RDT (cRDT); and Alere ${ }^{\mathrm{Tm}}$ malaria Ag Pf RDT (Alere), an ultrasensitive RDT (uRDT); were employed to detect $P$. falciparum infections. Both RDTs were based on the detection of P. falciparum HRP-2 antigen.

\section{Malaria diagnosis by nested PCR}

To detect parasite DNA, genomic DNA (gDNA) was isolated from the dried blood spots on filter paper using a QIAmp DNA Mini Kit (Qiagen, Hilden, Germany). Isolated genomic DNA was eluted into $35 \mu \mathrm{l}$ of elution buffer and used immediately or stored at $-20{ }^{\circ} \mathrm{C}$ until further use. For parasite detection, genus-specific nested PCR and species-specific nested PCR assays were performed using 


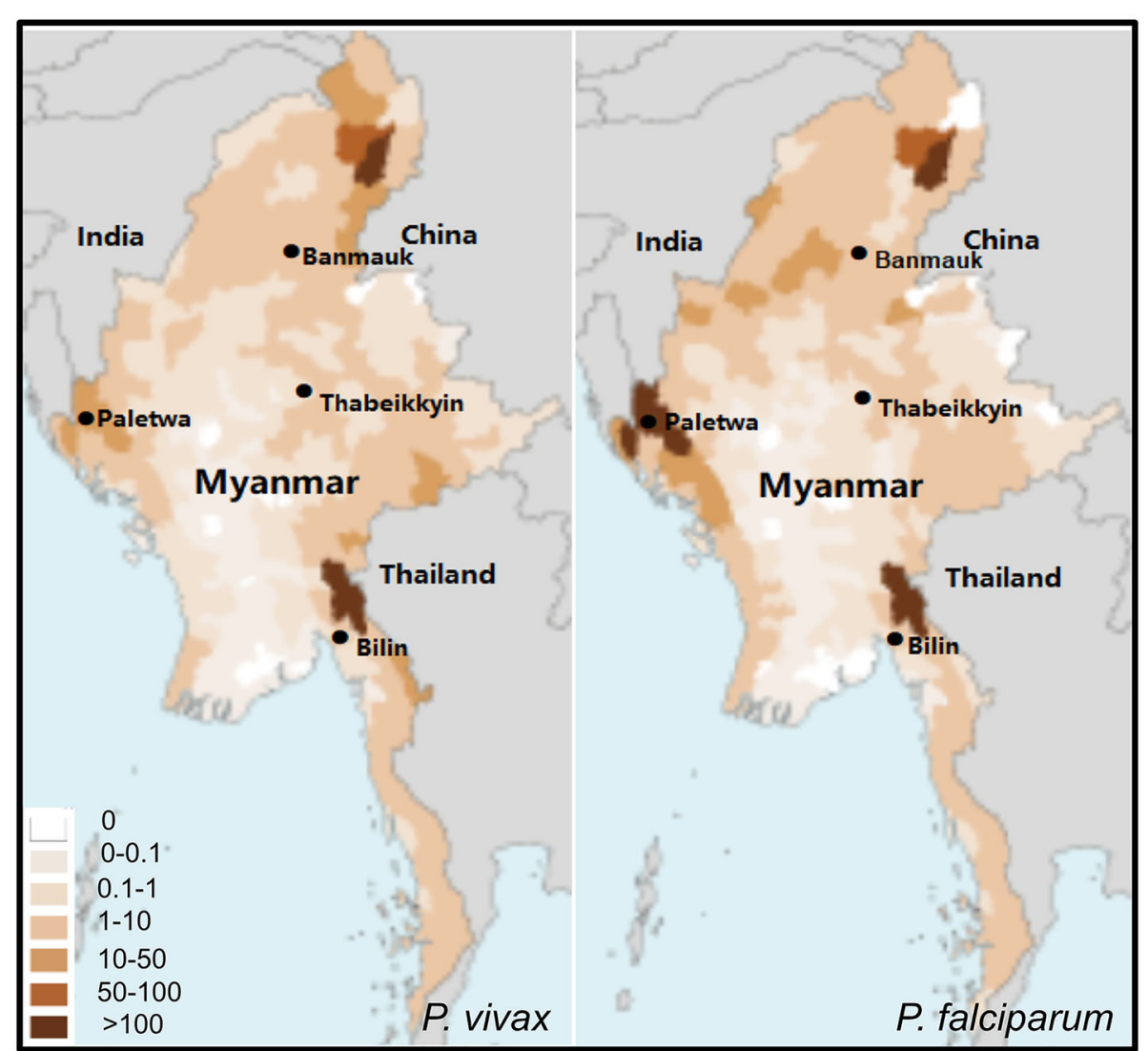

Fig. 1 Locations of the four townships where the cross-sectional surveys were conducted. Maps show the distribution of confirmed P. vivax (left) and P. falciparum (right) cases in Myanmar in 2016. Annual malaria incidences (confirmed cases per 1000 population) are color-coded

$2 \mu \mathrm{l}$ of gDNA and primers (see Additional file 1: Table S1) as described previously $[8,11,24,25]$.

\section{Sample pooling design}

For each positive sample identified by LM and/or RDT, species-specific nested PCR was performed to confirm the results. For the remaining negative samples, a pooling strategy was designed [19]. First, a small random set of 100 samples was selected and parasite prevalence was evaluated individually by nested PCR using Plasmodium genus-specific primers of rPLU1/rPLU5 (Nest1) and rPLU3/rPLU4 (Nest2). To design a pooling strategy for the remaining negative samples, a contingency table (Table 1) was generated to obtain an expected percent reduction of workload, $R_{\text {save }}$, for infection rate using the following formula: $R_{\text {save }}=$ $\mathrm{T}_{\text {save }} / \mathrm{T}_{\text {individual }} \times 100 \%=\left(2+\mathrm{P} \times \mathrm{n}_{1}-2 / \mathrm{N}-2 \mathrm{PN}-\mathrm{P} \times \mathrm{n} 2\right) /(2$ $\left.+\mathrm{P} \times \mathrm{n}_{1}\right), \mathrm{T}_{\text {individual }}=\mathrm{S} \times 2+\mathrm{P} \times \mathrm{S} \times \mathrm{n}_{1}=\mathrm{S} \times\left(2+\mathrm{P} \times \mathrm{n}_{1}\right), \mathrm{T}_{\text {pooling }}=(\mathrm{S} /$ $\mathrm{N}) \times 2+\mathrm{P} \times \mathrm{S} \times \mathrm{N} \times 2+\mathrm{P} \times \mathrm{S} \times \mathrm{n} 2=\mathrm{S} \times(2 / \mathrm{N}+2 \mathrm{P} \times \mathrm{N}+\mathrm{P} \times \mathrm{n} 2), \mathrm{T}_{\text {save }}=$ $\mathrm{T}_{\text {individual }}-\mathrm{T}_{\text {pooling }} \mathrm{S} \times\left(2+\mathrm{P} \times \mathrm{n}_{1}\right)-\mathrm{S} \times(2 / \mathrm{N}+2 \mathrm{PN}+\mathrm{P} \times \mathrm{n} 2)$ $=\mathrm{S} \times\left(2+\mathrm{P} \times \mathrm{n}_{1}-2 / \mathrm{N}-2 \mathrm{PN}-\mathrm{P} \times \mathrm{n} 2\right)$, where $\mathrm{T}_{\text {individual }}$ refers to the number of tests performed if the samples are tested individually, $\mathrm{T}_{\text {pooling }}$ the number of tests performed if the samples are pooled, and $\mathrm{T}_{\text {save }}$ the number of tests saved if the samples are pooled versus tested individually. In the formula, $\mathrm{P}$ is the estimated infection rate, $\mathrm{N}$ number of samples in each pool, $\mathrm{n}_{1}$ number of species ( 5 in this study), and $n_{2}$ the number of groups of the species are divided into for nested PCR ( 3 in this study). $R_{\text {save }}$ was estimated assuming that each pool contains no more than one positive sample. For each positive pool, samples were first tested individually by using genus-specific nested PCR (Fig. 2). For the positive samples, species-specific primers for $P$. falciparum, $P$. vivax, $P$. malariae, $P$. ovale and $P$. knowlesi were used to identify the Plasmodium species using primary PCR product as the template. Nested PCR for species were grouped into three reactions and multiplexed for P. falciparum $+P$. knowlesi and P. malariae $+P$. ovale based on easy separation of the PCR products. PCR products were separated in $1.2 \%$ agarose gel for visualization.

\section{Statistical analysis}

Data were analyzed by SPSS v.22.0. Malaria prevalence was calculated by crosstabs. The odds ratio (OR) was calculated for binary variables and Chi-square or Fisher's exact tests were used where appropriate. $P<0.05$ was 
Table 1 Percent reduction (\%) of workload for different extent of sample pooling (2-8 samples/pool) based on estimated infection rates (1-20\%)

\begin{tabular}{|c|c|c|c|c|c|c|c|}
\hline \multirow{2}{*}{$\begin{array}{l}\text { Estimated infection } \\
\text { rate (\%) }\end{array}$} & \multicolumn{7}{|c|}{ Number of samples per pool } \\
\hline & 2 & 3 & 4 & 5 & 6 & 7 & 8 \\
\hline 1 & 47.80 & 63.09 & 70.24 & 74.15 & 75.93 & 77.77 & 78.54 \\
\hline 2 & 45.71 & 59.68 & 65.71 & 68.57 & 69.37 & 70.20 & 70.00 \\
\hline 3 & 43.72 & 56.43 & 61.40 & 63.26 & 63.10 & 62.99 & 61.86 \\
\hline 4 & 41.82 & 53.33 & 57.27 & 58.18 & 57.12 & 56.10 & 54.09 \\
\hline 5 & 40.00 & 50.37 & 53.33 & 53.33 & 51.41 & 49.52 & 46.67 \\
\hline 6 & 38.26 & 47.54 & 49.57 & 48.70 & 45.94 & 43.23 & 39.57 \\
\hline 7 & 36.60 & 44.82 & 45.96 & 44.26 & 40.71 & 37.20 & 32.77 \\
\hline 8 & 35.00 & 42.22 & 42.50 & 40.00 & 35.69 & 31.43 & 26.25 \\
\hline 9 & 33.47 & 39.73 & 39.18 & 35.92 & 30.88 & 25.89 & 20.00 \\
\hline 10 & 32.00 & 37.33 & 36.00 & 32.00 & 26.27 & 20.57 & 14.00 \\
\hline 11 & 30.59 & 35.03 & 32.94 & 28.24 & 21.83 & 15.46 & 8.24 \\
\hline 12 & 29.23 & 32.82 & 30.00 & 24.62 & 17.56 & 10.55 & 2.69 \\
\hline 13 & 27.92 & 30.69 & 27.17 & 21.13 & 13.46 & 5.82 & -2.64 \\
\hline 14 & 26.67 & 28.64 & 24.44 & 17.78 & 9.51 & 1.27 & -7.78 \\
\hline 15 & 25.45 & 26.67 & 21.82 & 14.55 & 5.70 & -3.12 & -12.73 \\
\hline 16 & 24.29 & 24.76 & 19.29 & 11.43 & 2.02 & -7.35 & -17.50 \\
\hline 17 & 23.16 & 22.92 & 16.84 & 8.42 & -1.52 & -11.43 & -22.11 \\
\hline 18 & 22.07 & 21.15 & 14.48 & 5.52 & -4.94 & -15.37 & -26.55 \\
\hline 19 & 21.02 & 19.44 & 12.20 & 2.71 & -8.25 & -19.18 & -30.85 \\
\hline 20 & 20.00 & 17.78 & 10.00 & 0.00 & -11.44 & -22.86 & -35.00 \\
\hline
\end{tabular}

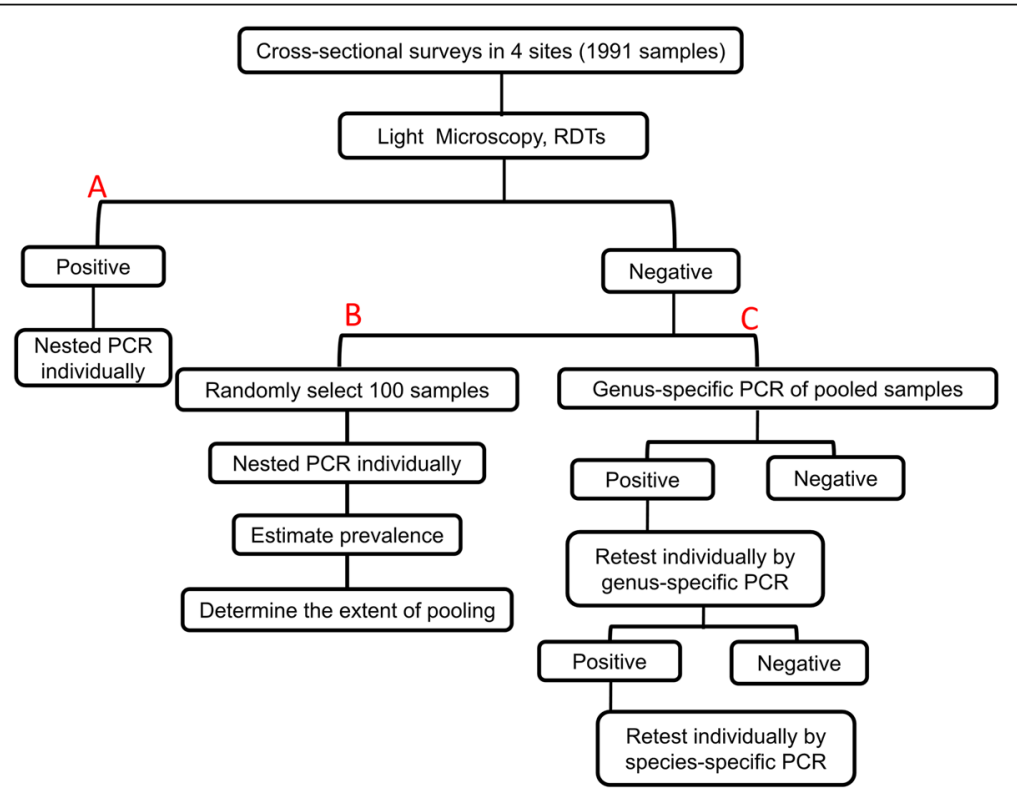

Fig. 2 Sample processing and assay work flow of the nested PCR pooling design. A: All samples positive by LM and RDTs were validated individually by nested PCR. B: Initial analysis of 100 random samples from the negatives by LM and RDT to estimate the infection prevalence and determine the degree of pooling. C: Pooling design of nested PCR of the remaining negative samples by LM and RDT 
considered statistically significant. The sensitivity and specificity of uRDT and cRDT in detecting slide-positive and nested PCR-positive P. falciparum infections in our study population were calculated by crosstabs with LM or nested PCR as the gold standard.

\section{Results \\ Geographical variations in the prevalence of malaria infections}

A total of 1991 participants were enrolled for the cross-sectional surveys of malaria prevalence: Bilin $(n=$ 315), Banmauk $(n=631)$, Thabeikkyin $(n=414)$ and Paletwa $(n=633)$. The overall parasite prevalence was $13.9 \%(277 / 1991)$ when all positive samples identified by at least one method were considered (Table 2). The limits of detection of the nested PCR was $0.1-1,0.1-1$, 1-5 and 1-5 parasites/ $\mu$ for $P$. falciparum, $P$. vivax, $P$. malariae and $P$. ovale, respectively [8]. As expected, nested PCR was the most sensitive and detected 226 (11.4\%) Plasmodium infections in the study populations. In stark contrast, LM only detected 13 P. falciparum and one for $P$. vivax, probably reflecting the low parasite densities associated with asymptomatic infections in low-endemicity regions. Screening by $P$. falciparum infections by cRDTs and uRDTs estimated parasite prevalences of 3.7\% (73/1991) and 5.0\% (100/1991), respectively. For parasite species, $P$. vivax accounted for the majority of the infections $69.9 \%$ (158/226), while P. falciparum and $P$. ovale were responsible for $25.2 \%(57 / 226)$ and $0.4 \%(1 / 226)$, respectively. Mixed infections by $P$. vivax/P. falciparum and $P$. vivax $/ P$. ovale accounted for $3.5 \%(8 / 226)$ and $0.9 \%(2 / 226)$, respectively (Fig. 3). Neither P. knowlesi nor P. malariae was identified.

The four townships had significantly different prevalences of malaria infections and parasite species compositions. Consistent with the malaria endemicity from confirmed malaria cases, Paletwa had the highest rate (22.9\%) of asymptomatic malaria infections, followed by Bilin and Banmauk ( 12\%), and Thabeikkyin had the lowest prevalence (3.9\%). LM only identified parasite infections in the western and northern townships, whereas it did not detect any in the eastern and central townships. In the southeastern and northern townships, $P$. vivax was the predominant species detected, accounting for $\sim 80 \%$ or higher proportions of infections, whereas the western township Paletwa still had a considerable proportion ( 34\%) of P. falciparum infections (Fig. 3). It is noteworthy that Bilin, considered malaria-free based on microscopy and RDTs, still had a substantial level (12.4\%) of asymptomatic vivax infections (Table 2, Fig. 3).

\section{Comparison of different methods in detecting $P$. falciparum infections}

Of the 1991 participants, 131 were diagnosed with P. falciparum infections by at least one method, of which $3.3 \%$ $(n=65), 0.7 \%(n=13), 3.7 \%(n=73)$ and $5.0 \%(n=100)$ were detected by nested PCR, LM, cRDT and uRDT, respectively. A Venn diagram shows the overlap in the number (percent) of individuals with $P$. falciparum infections detected by the four methods (Fig. 4). Of all the positive samples, only six participants were diagnosed with $P$. falciparum by all methods. For the $13 P$. falciparum positives determined by LM, 11, 8 and 7 were also detected by uRDT, cRDT and nested PCR, respectively, whereas two samples were failed by both nested PCR and RDTs. Of all $100 P$. falciparum positives by uRDT, 17 samples were negative by all the other detection methods (nested PCR, $\mathrm{LM}$ and cRDT). uRDT detected all samples that were positive by cRDT. Among 65 P. falciparum positives from nested PCR, only $10.8 \%(n=7), 55.4 \%(n=36)$ and $43.1 \%$ $(n=28)$ were also positive by LM, uRDT and cRDT, respectively, whereas $44.6 \%(n=29)$ was not detected by the rest of detection methods (LM, uRDT and cRDT).

The sensitivity and specificity of the two RDTs for detecting $P$. falciparum infections in the cross-sectional surveys were evaluated using LM or nested PCR as the gold standard (Table 3). Overall, the specificities of both RDTs were high (>95\%). When LM was used as reference, both RDTs showed modest sensitivities (84.6\% and 61.5\%). Their sensitivities were much lower (55.4\% and 43.1\%) when nested PCR was used as the gold standard. Clearly, uRDT had much higher sensitivities than cRDT and detected $20 \%$ more positive cases than cRDT.

Table 2 Malaria prevalence [number (\%)] in different townships

\begin{tabular}{|c|c|c|c|c|c|c|c|c|c|c|c|c|}
\hline \multirow[t]{2}{*}{ Townships } & \multirow{2}{*}{$\begin{array}{l}\text { No. of } \\
\text { participants } \\
\text { surveyed }\end{array}$} & \multirow[t]{2}{*}{ LM } & \multirow[t]{2}{*}{ cRDT } & \multirow[t]{2}{*}{ uRDT } & \multirow{2}{*}{$\begin{array}{l}\text { LM, RDT, } \\
\text { or both }\end{array}$} & \multicolumn{6}{|c|}{ Nested PCR } & \multirow[t]{2}{*}{ Total } \\
\hline & & & & & & $\overline{P V}$ & $P f$ & Po & $P v+P f$ & $P v+P o$ & Total & \\
\hline Bilin & 315 & 0 & 0 & 0 & 0 & 31 & 6 & 0 & 2 & 0 & 39 (12.4) & 39 (12.4) \\
\hline Banmauk & 631 & $3(0.5)$ & $5(0.8)$ & $16(2.5)$ & $18(2.9)$ & 56 & 11 & 0 & 0 & 0 & $67(10.6)$ & 77 (12.2) \\
\hline Thabeikkyin & 412 & $0(0)$ & $1(0.2)$ & $3(0.7)$ & $3(0.7)$ & 7 & 6 & 0 & 1 & 0 & $14(3.4)$ & $16(3.9)$ \\
\hline Paletwa & 633 & $11(1.7)$ & $67(10.6)$ & 81 (12.8) & $82(13.0)$ & 64 & 34 & 1 & 5 & 2 & $106(16.7)$ & 145 (22.9) \\
\hline Total & 1991 & $14(0.7)$ & $73(3.7)$ & $100(5.0)$ & $103(5.2)$ & 158 & 57 & 1 & 8 & 2 & $226(11.4)$ & 277 (13.9) \\
\hline
\end{tabular}

Abbreviations: $L M$ light microscopy, $C R D T$ conventional RDT, uRDT ultrasensitive RDT, Pv P. vivax, Pf P. falciparum, Po P. ovale 


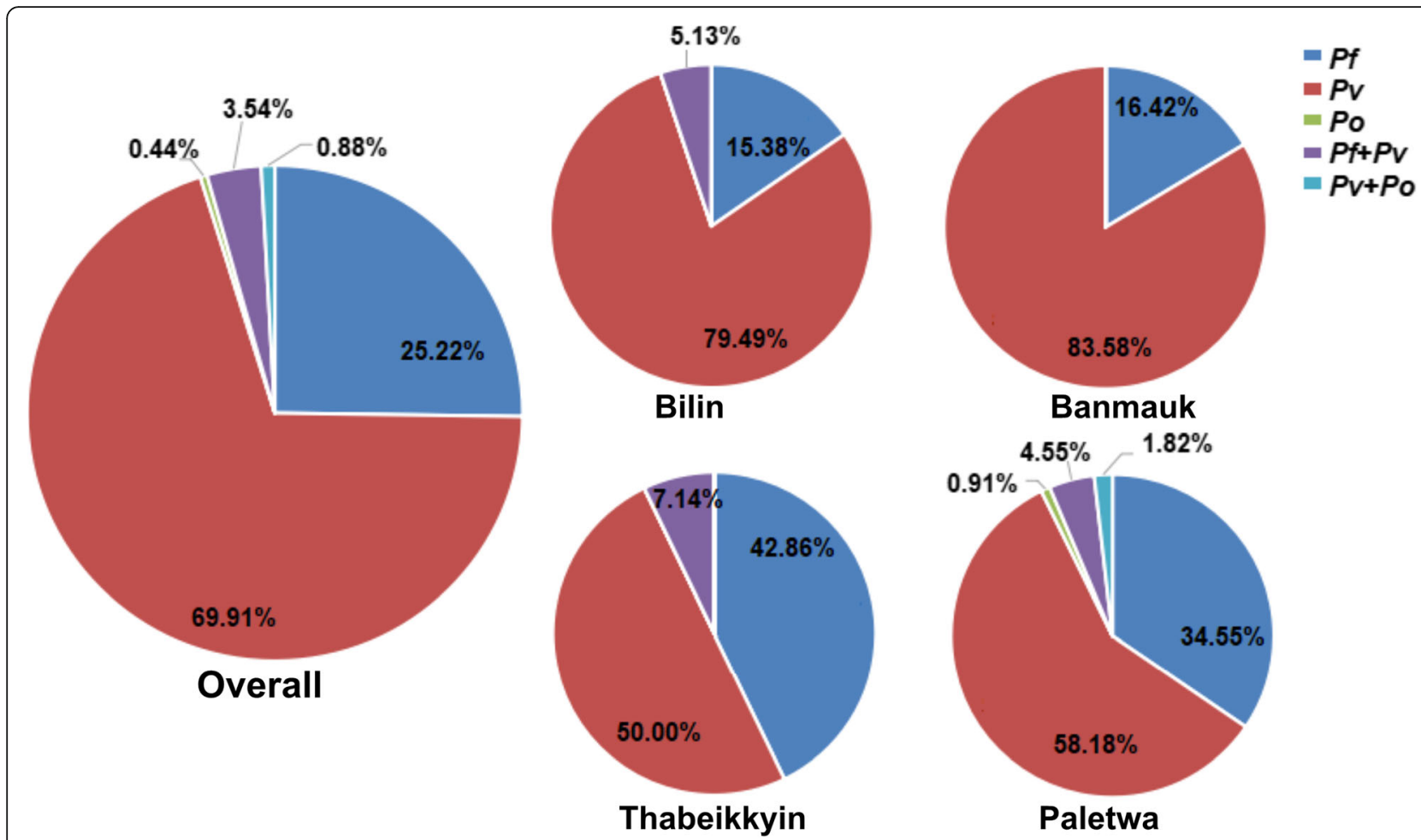

Fig. 3 Malaria species compositions in different study sites. The pie graphs showed the percentage of each species identified in four study areas and all populations. Abbreviations: Pf, P. falciparum; PV, P. vivax; Po, P. ovale

\section{Risk factors of subclinical malaria infections}

Because the visits to the surveyed villages were done in the daytime, when adults and men were probably away from home engaging in agricultural activities, the study population were slightly female-biased (56.91\%) and relatively young (median age: 14 years, range: 7 months to 90 years) (Table 4). Parasite prevalence was similar in both male and female populations, and also similar among the three age groups examined. Of the 1991 participants, 29 individuals had fever at the time of the

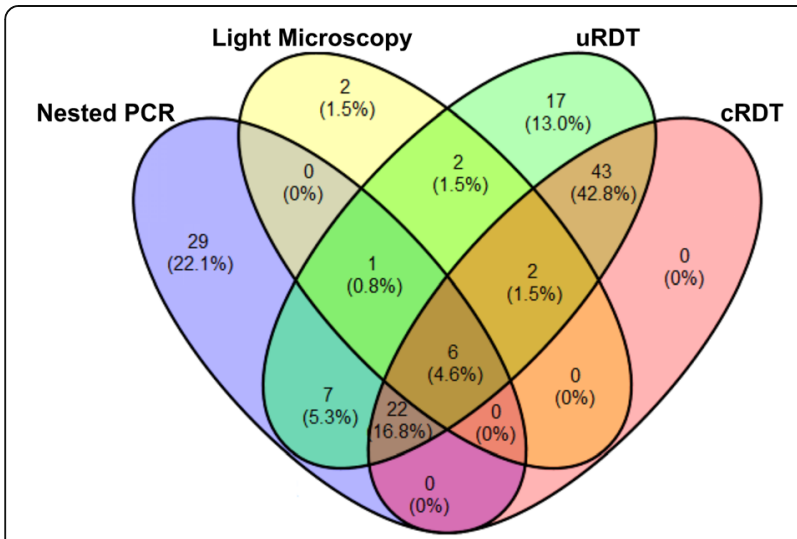

Fig. 4 Venn diagram showing the overlap in the number (percentage) of individuals with P. falciparum infections in 1991 volunteers as detected by LM, nested PCR, URDT and CRDT survey. Among them, 14 were infected with Plasmodium, but only five had a parasitemia that was high enough to be detected by LM and could be considered as having acute malaria episodes. By the binary logistic regression analysis, febrile participants had significantly higher prevalence and odds of malaria infection than the non-febrile $(\mathrm{OR}=7.51, P<0.001)$.

\section{Efficiency of the pooling strategy for molecular epidemiological studies}

To obtain highly accurate infection prevalence by molecular methods in low-endemic areas, sample pooling is necessary. Table 1 shows the calculated reduction of workload under different extents of pooling based on an estimated infection rate of $1-20 \%$. In a small set of 100 randomly selected negative samples by both LM and RDTs, nested PCR analysis identified 9\% Plasmodiumpositive samples. At this infection rate, having three samples per pool would give a maximum reduction of workload ( 40\%). Excluding 103 positive samples identified by LM and/or RDTs, 1788 samples were divided into 596 pools and genus-specific nested PCR identified 126 pools as positive. All samples from the 126 positive pools were amplified individually by genus-specific nested PCR (756 reactions), of which 158 were positive. To confirm parasite species, each of the 158 samples was re-tested in three reactions of species-specific PCR 
Table 3 Sensitivity and specificity of the two RDTs for detecting parasite infections

\begin{tabular}{|c|c|c|c|c|c|c|c|c|c|}
\hline \multirow[t]{2}{*}{ Gold standaro } & & \multicolumn{4}{|l|}{ uRDT } & \multicolumn{4}{|l|}{ CRDT } \\
\hline & & Positive $(n)$ & Negative $(n)$ & SE (\%) $(95 \% \mathrm{Cl})$ & SP (\%) $(95 \%$ Cl) & Positive $(n)$ & Negative $(n)$ & SE (\%) $(95 \%$ Cl) & SP (\%) $(95 \%$ Cl) \\
\hline \multirow[t]{2}{*}{ LM } & Positive & 11 & 2 & $84.6(61.9-100.0)$ & $95.5(94.6-96.4)$ & 8 & 5 & $61.5(30.9-92.1)$ & 96.7 (95.9-97.5) \\
\hline & Negative & 89 & 1889 & & & 65 & 1923 & & \\
\hline \multirow[t]{2}{*}{ Nested PCR } & Positive & 36 & 29 & $55.4(43.0-67.8)$ & 96.9 (95.9-97.5) & 28 & 37 & $43.1(30.7-55.4)$ & $97.7(97.0-98.3)$ \\
\hline & Negative & 64 & 1862 & & & 45 & 1881 & & \\
\hline
\end{tabular}

Abbreviations: SE sensitivity, SP specificity, $95 \% \mathrm{Cl} 95 \%$ confidence interval

(total 474 reactions). With this pooling design, a total of 2170 PCR reactions were performed as compared to 4050 if all samples had been analyzed individually $(1788 \times 2+158 \times 3)$. In another word, this pooling strategy reduced the workload by $46.4 \%$.

\section{Discussion}

It is believed that low-density asymptomatic infections are expected to be common in high-endemic areas with high herd immunity and premunition in the population [26]. Contrary to this conventional belief, asymptomatic infections are also found to be prevalent in areas of low endemicity [27]. A recent epidemiological study in Cambodia, Vietnam and along the Thailand-Myanmar border showed that approximately $20 \%$ of the population in these settings harbored $P$. falciparum or $P$. vivax infections, most of which were afebrile and asymptomatic [28]. Similarly, this report found 3.9-22.9\% of the residents in four malaria endemic areas carrying Plasmodium infections. Compared with the confirmed acute malaria infections reported in 2017, the different prevalence of subclinical infections in these endemic areas appeared to correspond to the levels of acute malaria and very well reflected the local malaria transmission intensities. The majority of the asymptomatic infections were present at parasite densities below the detection limit of LM. Such submicroscopic infections could develop into much higher-density infections at a later time and cause acute diseases [29, 30]. In addition, they are capable of infecting mosquitoes and contributing to transmission [31-33]. Therefore, low-density asymptomatic malaria parasitemia could influence transmission dynamics and sustain malaria endemicity, which might undermine elimination efforts [34]. Asymptomatic infections need to be closely monitored and dealt with adequate control measures in regions pursuing malaria elimination.

This study identified significant heterogeneity in the prevalence of subclinical malaria infections in different geographical regions of Myanmar, a country with the heaviest malaria burden in the GMS. The prevalence of Plasmodium infections in the four townships surveyed ranged from 3.9 to $22.9 \%$. Paletwa, located in the south-west site of Chin State had the highest parasite prevalence. Its mountainous terrain and poor accessibility thwart malaria control efforts. Bordering malarious areas of Bangladesh and India further promotes travel-associated malaria and malaria introduction [35]. Interestingly, the study population in Bilin had no detectable parasite infections by LM and RDTs, but nested PCR analysis revealed a substantial level of submicroscopic infection. This geographical heterogeneity indicates that malaria control strategy needs to be tailored to specific regions with different malaria epidemiology. While proper case management continues to be a focus of control efforts, novel strategies are needed to target the silent parasite reservoirs.

This study assessed several available malaria diagnostic tools for screening asymptomatic malaria infections. LM examination, the traditional gold standard for diagnosis of clinical malaria, performed poorly in this study with only 14 Plasmodium infections detected, accounting for $5 \%(14 / 277)$ of total infections identified by other methods. This is likely due to the low parasite densities associated with the asymptomatic infections, especially by $P$. vivax. Therefore, the use of microscopy is likely to

Table 4 Study population demographics and risk analysis

\begin{tabular}{|c|c|c|c|c|c|}
\hline & & $N(\%)$ & Prevalence, $n(\%)$ & OR $(95 \% \mathrm{Cl})$ & $P$-value \\
\hline \multirow[t]{2}{*}{ Sex } & Female & $1133(56.9)$ & $126(11.1)$ & 1 & \\
\hline & Male & $858(43.1)$ & $105(12.2)$ & $1.11(0.85-1.47)$ & 0.441 \\
\hline \multirow[t]{2}{*}{ Fever } & No & $1962(98.5)$ & $217(11.1)$ & 1 & \\
\hline & Yes & $29(1.5)$ & $14(48.3)$ & $7.51(3.57-15.76)$ & $<0.0001$ \\
\hline \multirow[t]{3}{*}{ Age groups (years) } & $\leq 5$ & $228(11.5)$ & 29 (12.7) & $1.15(0.74-1.78)$ & 0.544 \\
\hline & $6-14$ & $814(40.9)$ & $95(11.7)$ & $1.04(0.78-1.40)$ & 0.801 \\
\hline & $\geq 15$ & $949(47.7)$ & $107(11.3)$ & 1 & \\
\hline
\end{tabular}


result in a gross underestimation of the malaria burden [36]. RDTs for detection of Plasmodium antigens are commonly used for malaria diagnosis due to its simplicity. Conventional RDTs have similar detection limits as microscopy, but uRDTs may be ten times more sensitive than cRDTs. This study showed that uRDTs identified $20 \%$ more $P$. falciparum infections. However, one limitation for the $P$. falciparum RDTs is the high prevalence of $P$. vivax infections in the GMS, and uRDTs to detect $P$. vivax are not available. Moreover, other disadvantages of HRP-2 based RDTs are the presence of P. falciparum parasites with hrp2 gene deletion in the study region (cause of false negatives) [25], and continuous circulation of HRP-2 antigen long after successful treatment of infections (cause of false positives). The latter would exaggerate the rate of infections at the time of the surveys and is probably the major reason for the discrepancy in detection results between RDTs and nested PCR in our study. PCR is the method of choice for epidemiological studies of malaria, but the high cost of labor and reagents prevents its use for large-scale screening purposes. To make it more practical for large-scale epidemiological studies, a pooling strategy would be more cost effective $[8,18]$. A pooling strategy is further developed in this study, where the degree of sample pooling is optimized based on estimated prevalence of infections, which can be determined by studying a small, random subset of samples. Given the changing malaria epidemiology this step is necessary, especially for areas where the prevalence is under $10 \%$ [37-39]. In this study, with an estimated parasite infection rate of $9 \%$, the pooling nested PCR reduced the workload by $46.4 \%$.

\section{Conclusions}

This study shows that large proportions of individuals in malaria endemic areas of Myanmar carried subclinical malaria infections with pronounced geographical heterogeneity. In all four townships, $P$. vivax was the predominant species. In the western border township $P$. falciparum was also substantially prevalent. Whereas LM was deemed not suitable for detecting subclinical infections in these low-endemicity areas, the nested PCR method incorporating a pooling strategy would enable the implementation of molecular epidemiology studies on a large scale. Such information is important to decision-makers for future plans in the national malaria elimination program.

\section{Additional file}

Additional file 1: Table S1. Primers for pooling strategy of nested PCR (Plasmodium spp. detection). (DOCX $15 \mathrm{~kb}$ )

\section{Abbreviations}

Cl: Confidence interval; CRDT: Conventional RDT; LM: Light microscopy; OR: Odds ratio; PCR: Polymerase chain reaction; RDTs: Rapid diagnostic tests; URDT: Ultrasensitive RDT

\section{Acknowledgments}

We thank all the participants for their willingness to take part in this study. We are indebted to the technicians, nurses and other workers from local hospitals for their assistance to complete the field surveys.

\section{Funding}

This study was supported by a grant from the National Institute of Allergy and Infectious Diseases, National Institutes of Health (2U19Al089672) and Gates Foundation through Alere Standard Diagnostic Company.

\section{Availability of data and materials}

Data supporting the conclusions of this article are included within the article and its additional files. The datasets used and/or analyzed during the present study are available from the corresponding authors upon request.

\section{Authors' contributions}

ZL performed the laboratory study and drafted the manuscript. AT, TS, CC, $P A, M K$ and $Y Z$ participated in field work. ZL, TS, ZY, YL, HY, LW, XL, DL, ZP $J W, Y L$ and $Y Z$ participated in data analysis. QW, MK, YC and LC conceived the study and revised the manuscript. All authors read and approved the final manuscript.

\section{Ethics approval and consent to participate}

The study protocol was reviewed and approved by the institutional review boards of the Ministry of Health and Sports, Myanmar; China Medical

University, China; and Pennsylvania State University, USA. Before carrying out the study, all adult participants or legal guardians of children voluntarily signed the informed consent.

\section{Consent for publication}

Not applicable.

\section{Competing interests}

The authors declare that they have no competing interests.

\section{Publisher's Note}

Springer Nature remains neutral with regard to jurisdictional claims in published maps and institutional affiliations.

\section{Author details}

'Department of Immunology, College of Basic Medical Sciences, China Medical University, Shenyang 110122, Liaoning, China. ²Department of Clinical Laboratory, Baotou central Hospital, Baotou 014040, Inner Mongolia Province, China. ${ }^{3}$ Department of Public Health, Ministry of Health and Sports, Nay Pyi Taw, Myanmar. ${ }^{4}$ Myanmar Medical Association, Yangon, Myanmar. ${ }^{5}$ Department of Pathogen Biology and Immunology, Kunming Medical University, Kunming 650000, China. ${ }^{6}$ Yunnan Provincial Center of Arborvirus Research, Yunnan Provincial Key Laboratory of Vector-borne Diseases Control and Research, Yunnan Institute of Parasitic Diseases, Pu'er 665000, Yunnan, China. ${ }^{7}$ Department of Internal Medicine, Morsani College of Medicine, University of South Florida, 3720 Spectrum Boulevard, Suite 304, Tampa, FL 33612, USA.

Received: 16 August 2018 Accepted: 31 January 2019

Published online: 18 February 2019

\section{References}

1. WHO. World Malaria Report 2018. Geneva: World Health Organization. https://www.who.int/malaria/publications/world-malaria-report-2018/en/. Accessed 19 Nov 2018.

2. WHO. Strategy for malaria elimination in the Greater Mekong Subregion (2015-2030). Geneva: World Health Organization. http://apps.who.int/iris/ rest/bitstreams/923075/retrieve. Accessed 22 May 2015.

3. Lindblade KA, Steinhardt L, Samuels A, Kachur SP, Slutsker L. The silent threat: asymptomatic parasitemia and malaria transmission. Expert Rev Anti Infect Ther. 2013;11:623-39.

4. Huang F, Takala-Harrison S, Liu H, Xu JW, Yang HL, Adams M, et al. Prevalence of clinical and subclinical Plasmodium falciparum and Plasmodium vivax malaria in two remote rural communities on the Myanmar-China border. Am J Trop Med Hyg. 2017;97:1524-31. 
5. Zaw MT, Thant M, Hlaing TM, Aung NZ, Thu M, Phumchuea K, et al. Asymptomatic and sub-microscopic malaria infection in Kayah State, eastern Myanmar. Malar J. 2017;16:138.

6. Ghinai I, Cook J, Hla TT, Htet HM, Hall T, Lubis IN, et al. Malaria epidemiology in central Myanmar: identification of a multi-species asymptomatic reservoir of infection. Malar J. 2017:16:16.

7. Mfueni E, Devleesschauwer B, Rosas-Aguirre A, Van Malderen C, Brandt PT, Ogutu B, et al. True malaria prevalence in children under five: Bayesian estimation using data of malaria household surveys from three sub-Saharan countries. Malar J. 2018:17:65

8. Wang B, Han SS, Cho C, Han JH, Cheng Y, Lee SK, et al. Comparison of microscopy, nested-PCR, and real-time-PCR assays using high-throughput screening of pooled samples for diagnosis of malaria in asymptomatic carriers from areas of endemicity in Myanmar. J Clin Microbiol. 2014;52: 1838-45.

9. Thakor HG. Laboratory diagnosis of malaria. J Indian Med Assoc. 2000;98:623-7.

10. Kain KC, Harrington MA, Tennyson S, Keystone JS. Imported malaria: prospective analysis of problems in diagnosis and management. Clin Infect Dis. 1998;27:142-9.

11. Snounou G, Viriyakosol S, Zhu XP, Jarra W, Pinheiro L, do Rosario VE, et al. High sensitivity of detection of human malaria parasites by the use of nested polymerase chain reaction. Mol Biochem Parasitol. 1993;61:315-20.

12. Humar A, Ohrt C, Harrington MA, Pillai D, Kain KC. Parasight F test compared with the polymerase chain reaction and microscopy for the diagnosis of Plasmodium falciparum malaria in travelers. Am J Trop Med Hyg. 1997;56:44-8.

13. Kattenberg $\mathrm{JH}$, Tahita CM, Versteeg IA, Tinto H, Traore Coulibaly M, D'Alessandro $U$, et al. Evaluation of antigen detection tests, microscopy, and polymerase chain reaction for diagnosis of malaria in peripheral blood in asymptomatic pregnant women in Nanoro, Burkina Faso. Am J Trop Med Hyg. 2012;87:251-6.

14. Parajuli K, Hanchana S, Inwong M, Pukrittayakayamee S, Ghimire P. Comparative evaluation of microscopy and polymerase chain reaction (PCR) for the diagnosis in suspected malaria patients of Nepal. Nepal Med Coll J. 2009:11:23-7.

15. Emmanuel JC, Bassett MT, Smith HJ, Jacobs JA. Pooling of sera for human immunodeficiency virus (HIV) testing: an economical method for use in developing countries. J Clin Pathol. 1988;41:582-5.

16. Babu PG, Saraswathi NK, Vaidyanathan H, John TJ. Reduction of the cost of testing for antibody to human immunodeficiency virus, without losing sensitivity, by pooling sera. Indian J Med Res. 1993;97:1-3.

17. Garcia Z, Taylor L, Ruano A, Pavon L, Ayerdis E, Luftig RB, et al. Evaluation of a pooling method for routine anti-HCV screening of blood donors to lower the cost burden on blood banks in countries under development. J Med Virol. 1996;49:218-22.

18. Westreich DJ, Hudgens MG, Fiscus SA, Pilcher CD. Optimizing screening for acute human immunodeficiency virus infection with pooled nucleic acid amplification tests. J Clin Microbiol. 2008;46:1785-92.

19. Taylor SM, Juliano JJ, Trottman PA, Griffin JB, Landis SH, Kitsa P, et al. High-throughput pooling and real-time PCR-based strategy for malaria detection. J Clin Microbiol. 2010;48:512-9.

20. WHO. Malaria country Profiles-Myanmar. Geneva: World Health Organization; 2017. https://www.who.int/malaria/publications/country-profiles/profile_ mmr_en.pdf. Accessed 29 Nov 2017.

21. WHO. Malaria Microscopy Standard Operating Procedures 2016. Geneva: World Health Organization; 2016.

22. WHO. Basic laboratory methods in medical parasitology. Geneva: World Health Organization; 1991.

23. Starzengruber P, Fuehrer HP, Ley B, Thriemer K, Swoboda P, Habler VE, et al. High prevalence of asymptomatic malaria in south-eastern Bangladesh. Malar J. 2014;13:16

24. Fuehrer HP, Stadler MT, Buczolich K, Bloeschl I, Noedl H. Two techniques for simultaneous identification of Plasmodium ovale curtisi and Plasmodium ovale wallikeri by use of the small-subunit rRNA gene. J Clin Microbiol. 2012;50:4100-2

25. Yan J, Li N, Wei X, Li P, Zhao Z, Wang L, et al. Performance of two rapid diagnostic tests for malaria diagnosis at the China-Myanmar border area. Malar J. 2013;12:73.

26. Bjorkman $\mathrm{AB}$. Asymptomatic low-density malaria infections a parasite survival strategy? Lancet Infect Dis. 2018;18:485-6.
27. Galatas B, Bassat Q, Mayor A. Malaria parasites in the asymptomatic. Looking for the hay in the haystack. Trends Parasitol. 2016;32:296-308.

28. Imwong M, Nguyen TN, Tripura R, Peto TJ, Lee SJ, Lwin KM, et al. The epidemiology of subclinical malaria infections in south-east Asia: findings from cross-sectional surveys in Thailand-Myanmar border areas, Cambodia, and Vietnam. Malar J. 2015;14:381.

29. Rogier C, Commenges D, Trape JF. Evidence for an age-dependent pyrogenic threshold of Plasmodium falciparum parasitemia in highly endemic populations. Am J Trop Med Hyg. 1996;54:613-9.

30. Nguyen TN, von Seidlein L, Nguyen TV, Truong PN, Hung SD, Pham HT, et al. The persistence and oscillations of submicroscopic Plasmodium falciparum and Plasmodium vivax infections over time in Vietnam: an open cohort study. Lancet Infect Dis. 2018;18:565-72.

31. Schneider P, Bousema JT, Gouagna LC, Otieno S, van de Vegte-Bolmer M, Omar SA, et al. Submicroscopic Plasmodium falciparum gametocyte densities frequently result in mosquito infection. Am J Trop Med Hyg. 2007;76:470-4.

32. Coleman RE, Kumpitak C, Ponlawat A, Maneechai N, Phunkitchar V, Rachapaew N, et al. Infectivity of asymptomatic Plasmodium-infected human populations to Anopheles dirus mosquitoes in western Thailand. J Med Entomol. 2004;41:201-8.

33. Kiattibutr K, Roobsoong W, Sriwichai $P$, Saeseu T, Rachaphaew N, Suansomjit C, et al. Infectivity of symptomatic and asymptomatic Plasmodium vivax infections to a Southeast Asian vector, Anopheles dirus. Int J Parasitol. 2017:47:163-70.

34. Bousema T, Okell L, Felger I, Drakeley C. Asymptomatic malaria infections: detectability, transmissibility and public health relevance. Nat Rev Microbiol. 2014;12:833-40.

35. Nguitragool W, Mueller I, Kumpitak C, Saeseu T, Bantuchai S, Yorsaeng R, et al. Very high carriage of gametocytes in asymptomatic low-density Plasmodium falciparum and P. vivax infections in western Thailand. Parasit Vectors. 2017;10:512

36. Okell LC, Ghani AC, Lyons E, Drakeley CJ. Submicroscopic infection in Plasmodium falciparum-endemic populations: a systematic review and meta-analysis. J Infect Dis. 2009;200:1509-17.

37. Nzobo BJ, Ngasala BE, Kihamia CM. Prevalence of asymptomatic malaria infection and use of different malaria control measures among primary school children in Morogoro Municipality, Tanzania. Malar J. 2015:14:491.

38. Koepfli C, Ome-Kaius M, Jally S, Malau E, Maripal S, Ginny J, et al. Sustained malaria control over an 8-year period in Papua New Guinea: the challenge of low-density asymptomatic Plasmodium infections. J Infect Dis. 2017;216:1434-43.

39. Niang M, Thiam LG, Sane R, Diagne N, Talla C, Doucoure S, et al. Substantial asymptomatic submicroscopic Plasmodium carriage during dry season in low transmission areas in Senegal: implications for malaria control and elimination. PLoS One. 2017;12:e0182189.
Ready to submit your research? Choose BMC and benefit from:

- fast, convenient online submission

- thorough peer review by experienced researchers in your field

- rapid publication on acceptance

- support for research data, including large and complex data types

- gold Open Access which fosters wider collaboration and increased citations

- maximum visibility for your research: over $100 \mathrm{M}$ website views per year

At $\mathrm{BMC}$, research is always in progress.

Learn more biomedcentral.com/submissions 